\title{
Blood pressure variability and medial temporal atrophy in apolipoprotein $\epsilon 4$ carriers
}

\author{
Isabel J. Sible ${ }^{1}$. Daniel A. Nation ${ }^{2,3}$ (D) for the Alzheimer's Disease Neuroimaging Initiative
}

Accepted: 31 August 2021 / Published online: 28 September 2021

(c) The Author(s) 2021

\begin{abstract}
Blood pressure variability is an emerging risk factor for dementia but relationships with markers of neurodegeneration and Alzheimer's disease risk are understudied. We investigated blood pressure variability over one year and follow-up medial temporal brain volume change in apolipoprotein $€ 4$ carriers and non-carriers, and in those with and without Alzheimer's disease biomarker abnormality. 1051 Alzheimer's Disease Neuroimaging Initiative participants without history of dementia or stroke underwent 3-4 blood pressure measurements over 12 months and $\geq 1$ MRI thereafter. A subset $(n=252)$ underwent lumbar puncture to determine Alzheimer's disease cerebral spinal fluid amyloid-beta and phosphorylated tau biomarker abnormality. Blood pressure variability over 12 months was calculated as variability independent of mean. Longitudinal hippocampal and entorhinal cortex volume data were extracted from serial brain MRI scans obtained after the final blood pressure measurement. Apolipoprotein $€ 4$ carrier status was defined as at least one $\epsilon 4$ allele. Bayesian growth modelling revealed a significant interaction of blood pressure variability by $\epsilon 4$ by time on hippocampal ( $B:-2.61$ [95\% credible interval $-3.02,-2.12]$ ) and entorhinal cortex ( $(3:-1.47$ [95\% credible interval -1.71, -1.17]) volume decline. A similar pattern emerged in subsets with Alzheimer's disease pathophysiology (i.e., abnormal levels of both amyloid-beta and phosphorylated tau). Findings suggest that elevated blood pressure variability is related to medial temporal volume loss specifically in $€ 4$ carriers, and in those with Alzheimer's disease biomarker abnormality. Findings could implicate blood pressure variability in medial temporal neurodegeneration observed in older $€ 4$ carriers and those with prodromal Alzheimer's disease.
\end{abstract}

Keywords Blood pressure variability; Medial temporal lobes; Apolipoprotein є4; Alzheimer’s disease; Biomarkers

Data used in preparation of this article were obtained from the Alzheimer's Disease Neuroimaging Initiative (ADNI) database (adni.loni.usc.edu). As such, the investigators within the ADNI contributed to the design and implementation of ADNI and or provided data but did not participate in analysis or writing of this report. A complete listing of ADNI investigators can be found at: http://adni.loni.usc.edu/wp-content/uploads/how_to_apply/ADNI_ Acknowledgement_List.pdf

Daniel A. Nation

dnation@uci.edu

1 Department of Psychology, University of Southern California, Los Angeles, CA 90007, USA

2 Institute for Memory Impairments and Neurological Disorders, University of California Irvine, Irvine, CA 92697, USA

3 Department of Psychological Science, University of California Irvine, Irvine, CA 92697, USA

\section{Introduction}

Blood pressure (BP) is among the most studied vascular risk factors linked to cognitive impairment, neuropathological change, and dementia (Lane et al., 2019; Zlokovic, 2011). There has been substantial work to determine the relationships between both high and low BP and brain pathology and cognitive outcomes, as well as the aggregate impact of dysregulated BP on later brain health (Lane et al., 2019). A recent randomized controlled trial found that aggressive BP lowering was related to a decreased incidence of cognitive impairment (Wright et al., 2015), suggesting a causal association between average BP levels and cognitive decline (Yaffe, 2019).

Beyond average levels, BP variability (BPV) over months and years represents an understudied aspect of BP as it relates to brain health (Yoo et al., 2020). Given the substantial overlap between vascular and Alzheimer's disease (AD) pathologies in the brains of individuals diagnosed with 
dementia (Schneider et al., 2007), there is growing interest to study BPV in the context of cognitive aging and AD risk. Recent work suggests that elevated BPV is associated with cerebrovascular disease (Ma, et al., 2020a) and predictive of cognitive impairment and dementia, including $\mathrm{AD}$ and vascular dementia (Ma et al., 2020b; Rouch et al., 2020; Yoo et al., 2020), even in healthy older adults with wellcontrolled average BP (Cho et al., 2018). Chronic large fluctuations in BP may stress arterial walls and promote microvascular injury and arterial remodeling (Nagai et al., 2017). These vascular changes may convey vulnerability to cerebral hypoperfusion injury (Sible et al., 2021b) and subsequent neuronal injury, especially in regions highly sensitive to BPrelated hypoxic-ischemic injury, such as the hippocampus (Iadecola, 2004; Ma et al,. 2020b; Vikner et al., 2021).

Consistent with this hypothesis, higher BPV has been linked to lower hippocampal volume in both cross-sectional (Sabayan et al., 2013) and longitudinal (Ma, et al. 2020b) studies of older adults without dementia. However, less is known about the relationship between BPV and brain volumes in other key regions of $\mathrm{AD}$, such as the entorhinal cortex. It is also unclear how BPV may be related to medial temporal atrophy rates in those at risk for AD due to the presence of the apolipoprotein 64 (APOE $€ 4$ ) gene, which has been associated with neurodegeneration and neurovascular deficits in the hippocampus and parahippocampal cortex (Burggren et al., 2008; Palop \& Mucke, 2011). Finally, while most studies of BPV in aging have drawn from samples clinically determined to be without history of dementia, a recent study found that BPV was increased in individuals with mild cognitive impairment (MCI) and AD biomarker abnormality (Sible et al., 2020). How these relationships may appear in samples with abnormal AD biomarkers is less known, and could have implications for neurodegeneration in $\mathrm{AD}$. The aims of the present study were to investigate the interactive relationship between BPV and APOE $€ 4$ carrier status in relation to hippocampal and entorhinal cortex volumetric change in older adults at risk for $\mathrm{AD}$, and to determine whether these relationships remained evident in those with ongoing $\mathrm{AD}$ pathophysiology (i.e., $\mathrm{AD}$ biomarker abnormality).

\section{Methods}

\section{Study design}

\section{Participants}

Data were obtained from the Alzheimer's Disease Neuroimaging Initiative (ADNI) database. The ADNI is a multisite natural history study that has collected clinical, biomarker, and neuropsychological data since 2003 to measure the progression of typical aging, MCI, and AD. Adults aged 55-91 were enrolled if they met the following criteria: few depressive symptoms (Geriatric Depression Scale $<6$ ), free of history of neurological disease (other than suspected AD), no greater than mild dementia symptoms (Clinical Dementia Rating scale $\leq 1$ ), and low vascular risk (Hachinski Ischemic Score $\leq 4)$. Ethical approval was obtained for each institution involved and all participants provided written informed consent. Further study details can be found online (https:// adni.loni.usc.eduL.

The present study included participants who underwent clinical evaluation at study baseline and BP measurement at study screening, baseline, and 6- and 12- months followup. Participants also underwent $\geq 1$ structural MRI after the 12-month follow-up BP collection. A subset underwent lumbar puncture to determine cerebral spinal fluid (CSF) AD biomarker levels.

\section{Measures}

\section{Clinical assessment}

Baseline clinical evaluation identified participants to be cognitively normal $(\mathrm{CN})$ or MCI. All participants were without history of dementia or stroke. MCI diagnostic criteria included (Petersen et al., 2010): subjective memory complaint; Mini Mental State Exam scores between 24 and 30 (inclusive); global Clinical Dementia Rating scale score of 0.5; scores on delayed recall of Story A of the Wechsler Memory Scale Revised Logical Memory II subtest that are below expected performance based on years of education; general presentation that would disqualify for a diagnosis of AD. Participants were categorized as $\mathrm{CN}$ if diagnostic criteria for MCI were not met. CN and MCI participants were then collapsed into one category of older adults without history of dementia or stoke and were used in all analyses.

\section{CSF AD biomarker assessment}

Baseline lumbar puncture and CSF analysis in a subset of participants determined amyloid-beta $(\mathrm{A} \beta)$ and phosphorylated tau (Ptau) levels as described elsewhere (Bittner et al., 2016; Hansson et al., 2018; Seibyl et al., 2017; Shaw et al., 2016). Using established guidelines, CSF A $\beta$ levels $\leq 980 \mathrm{pg} / \mathrm{mL}$ and CSF Ptau levels $\geq 21.8 \mathrm{pg} / \mathrm{mL}$ were considered abnormal (Hansson et al., 2018; Shaw et al., 2018).

Participants were then further categorized based on abnormal levels of both $\mathrm{A} \beta$ and Ptau $(\mathrm{A} \beta+\mathrm{Ptau}+)$, thus representing a group of older adults without a history of dementia or stroke confirmed to have AD pathophysiology (Jack et al., 2018). To examine associations in subsets not meeting biomarker criteria for $\mathrm{AD}($ e.g., $\mathrm{A} \beta+\mathrm{Ptau}+)$, remaining 
participants with available CSF biomarker data were then categorized as those with 1) one abnormal biomarker and one normal biomarker $(\mathrm{A} \beta+\mathrm{Ptau}-$ or $\mathrm{A} \beta$-Ptau +$)$; or 2$)$ two normal biomarkers (A $\beta$-Ptau-) (see Supplementary Table 1).

\section{BP assessment}

Seated BP measurements were obtained from participants 3-4 times between study screening and 12-months followup using a calibrated mercury sphygmomanometer, as described elsewhere (Sible et al., 2020, 2021b). Intraindividual BPV was calculated for each participant using the 3-4 BP measurements collected over the 12 month period as variation independent of mean (VIM), a commonly used index of visit-to-visit BPV that is uncorrelated with average BP levels across visits (de Heus et al., 2019; Rothwell et al., 2010; Rouch et al., 2020; Sible et al., 2020, 2021a, 2021b). VIM was calculated as: $\mathrm{VIM}=\mathrm{SD} / \mathrm{mean}^{x}$, where the power $x$ was derived from non-linear curve fitting of BP SD against average BP using the nls package in $\mathrm{R}$ ( $\mathrm{R}$ Core Team, 2018), as described elsewhere (Rothwell et al., 2010; Yano, 2017). Baseline hypertension was determined from the total sample average systolic BP taken at study baseline. Given that systolic BPV and diastolic BPV were correlated and primary findings were similar, main findings focused on systolic BPV and diastolic BPV findings are reported in Supplementary Materials.

\section{Volumetric MRI change assessment}

Participants underwent $\geq 11.5 \mathrm{~T}$ or $3 \mathrm{~T}$ MRI after the final BP collection at 12-months follow-up. Image acquisition and processing details can be found online (http://adni. loni.usc.edu/methods/documents/mri-protocols/). Briefly, T1-weighted structural images were collected using either a 3D-MPRAGE or 3D IR-SPGR sequence. The following values from each of these MRI scans were extracted from the adnimerge dataset (Fischl, 2012; Reuter et al., 2012): total hippocampal volume, total entorhinal cortex volume, whole brain volume (sum of gray matter and white matter volumes), and total intracranial volume (TIV; sum of gray matter, white matter and CSF volumes). Volumes were determined using the FreeSurfer imaging suite as described elsewhere (http://adni.loni.usc.edu/methods/), a software with good test-retest reliability for volumetric segmentation within and across scanners (Brown et al., 2020).

\section{Other measurements}

Demographic and clinical information was determined from baseline clinical evaluation. Baseline body mass index (BMI) was calculated as weight ( $\mathrm{kg}$ ) / height (meters) squared. Determination of APOE $\notin 4$ carrier status was performed as previously described (Saykin et al., 2010) using blood samples from baseline venipuncture and participants were categorized as those with at least one APOE e4 allele versus those without. Vascular risk was determined from baseline clinical evaluation, as described elsewhere (D'Agostino et al., 1994; Nation et al., 2015; Sible et al., 2020). Participants were categorized as having low ( $\leq 1$ vascular risk factor) or high ( $\geq 2$ vascular risk factors) vascular risk (D'Agostino et al., 1994). History of smoking and dyslipidemia were also determined from clinical evaluation at baseline. Information about medication use was determined at study baseline. Participants were categorized as those taking antihypertensive medication (all classes) versus those who were not, and those taking antidementia agents versus those who were not.

\section{Statistical analysis}

Bayesian linear growth modelling using the brms package in $\mathrm{R}$ ( $\mathrm{R}$ Core Team, 2018) examined the role of BPV, APOE $\epsilon 4$ carrier status, and the passage of time on volumetric change in hippocampus and entorhinal cortex. Compared to repeated measures ANOVA, Bayesian linear growth modelling handles missing participant data and thus boosts statistical power, as well as accommodates varying time windows of measurement. All models specified random intercepts for participant, to account for individual variation in volumetric change, and fixed effects for BPV and APOE $€ 4$ carrier status to test for differences in volumetric change due to BPV and APOE $€ 4$ carrier status, respectively. Only MRIs acquired after the final BP measurement at 12-months follow-up were used in analyses to determine temporal order of any associations; therefore, passage of time for MRIs was calculated as months elapsed since BPV determination (e.g., after the last BP measurement was collected at 12-months follow-up) (range: 6 - 108 months) and then grand centered at 0 . In an attempt to replicate a previous finding linking BPV to hippocampal volume decline (Ma et al., 2020c), we first ran models investigating a BPV by time interaction on volumetric change in the hippocampus and entorhinal cortex. Next, because we expected volumetric change might have different trajectories based on both BPV (Ma et al., 2020c) and APOE $€ 4$ carrier status (Palop \& Mucke, 2011), we additionally tested the interaction of BPV by APOE $\epsilon 4$ carrier status by time. We also examined the three-way interaction of BPV by APOE $\epsilon 4$ carrier status by time on volumetric change in exploratory post-hoc analyses of subsets not meeting biomarker criteria for AD (see Supplementary Table 2). Potential confounding variables were included in all models: age at MRI, sex, years of education, APOE $€ 4$ carrier status (for main effect models), TIV at MRI, baseline hypertension, antihypertensive medication use and vascular 
risk. Additional sensitivity analyses covaried for: 1) average BP, 2) history of smoking, 3) history of dyslipidemia, 4) use of antidementia agents, and 5) whole brain volume at MRI (instead of TIV). Supplementary analyses examined relationships between BPV and whole brain volume at MRI. All analyses were 2-tailed and effect estimates with credible intervals excluding 0 were considered significant.

\section{Results}

In primary analyses, 1051 participants without a history of dementia or stroke contributed to 2656 MRI scans (median 3 scans) and the median time interval between BPV measurement and MRI scan was 24 months (IQR: 30 months). In secondary analyses, 252 participants confirmed to have AD pathophysiology contributed to 595 MRI scans (median 3 scans) and the median time interval between BPV measurement and MRI scan was 12 months (IQR: 30 months). Table 1 and Supplementary Table 3 summarize baseline demographic and clinical information.

\section{$B P V$ and $A P O E \epsilon 4$ related to medial temporal volumetric change in older adults}

Primary analyses revealed a significant interaction of systolic BPV by time on hippocampal ( $B$ : -0.51 [95\% credible interval (CI) $-0.63,-0.30]$ ) and entorhinal cortex volume ( $B$ : -0.28 [95\% CI $-0.34,-0.22]$ ), indicating that participants with elevated systolic BPV were observed to have the fastest hippocampal and entorhinal cortex volume decline at follow-up (Fig. 1a). There was also a significant three-way interaction of systolic BPV by APOE $€ 4$ carrier status by time on hippocampal (B: -2.61 [95\% CI -3.02, -2.12]) and entorhinal cortex volume (B: -1.47 [95\% CI -1.71, -1.17]), suggesting that hippocampal and entorhinal cortex volume at follow-up decreased the fastest for APOE $€ 4$ carriers with elevated systolic BPV (Fig. 1b).

\section{$B P V$ and $A P O E \in 4$ related to medial temporal volumetric change in older adults with $A D$ biomarker abnormalities}

Secondary analyses of subsets with abnormal levels of both CSF A $\beta$ and Ptau also revealed a significant interaction of systolic BPV by time on hippocampal ( $\beta$ : -1.05 [95\% CI $-1.18,-0.36]$ ) and entorhinal cortex volume (B: - 0.78 [95\% CI -1.30, -0.56]), suggesting that hippocampal and entorhinal cortex volume change at follow-up was related to elevated systolic BPV in older adults confirmed to have AD pathophysiology (Fig. 2a). Additionally, there was a significant three-way interaction of systolic BPV by APOE $€ 4$ carrier status by time on hippocampal (B: - 1.89 [95\% CI -2.44,
Table 1 Baseline clinical and demographic information

\begin{tabular}{ll}
\hline & Total sample $(N=1051)$ \\
\hline Age (years) & $73.7(6.8)$ \\
Sex $(n, \%$ female) & $455(43.3 \%)$ \\
Education (years) & $16.0(2.8)$ \\
APOE $\epsilon 4$ carriers $(n, \%)$ & $437(41.6 \%)$ \\
MCI $(n, \%)$ & $680(64.7 \%)$ \\
A $\beta$ (n, \% abnormal) & $438(41.7 \%)$ \\
Ptau (n, \% abnormal) & $431(41.0 \%)$ \\
MMSE score & $28.1(1.7)$ \\
CDR-sb score & $0.96(0.96)$ \\
BMI (kg/m $\left.{ }^{2}\right)$ & $27.0(4.5)$ \\
Vascular risk $(n, \%$ low) & $978(93.1 \%)$ \\
Medication use $(n, \%)$ & \\
Antihypertensive agents & $439(41.8 \%)$ \\
ACE inhibitors & $181(17.2 \%)$ \\
ARBs & $96(9.1 \%)$ \\
Alpha blockers & $24(2.3 \%)$ \\
Calcium channel blockers & $82(7.8 \%)$ \\
Diuretics & $56(5.3 \%)$ \\
Antidementia agents & $398(37.9 \%)$ \\
Systolic BP (mmHg) &
\end{tabular}

Means and SDs shown unless otherwise indicated

MMSE, Mini Mental State Exam; $B P$, blood pressure; $B M I$, body mass index: VIM, variability independent of mean; $A P O E \epsilon 4$, apolipoprotein $\epsilon 4 ; M C I$, mild cognitive impairment; $C D R$-sb, Clinical Dementia Rating Scale sum of box score; $A \beta$, amyloid-beta; Ptau, phosphorylated tau; $A C E$ inhibitors, angiotensin-converting enzyme inhibitors; $A R B s$, angiotensin II receptor blockers

-1.31]) and entorhinal cortex ( $3:-1.36$ [95\% CI -2.04, -0.11]) volume, indicating that volumetric change at follow-up was related to elevated systolic BPV specifically in APOE $€ 4$ carriers with AD pathophysiology (Fig. 2b).

Exploratory post-hoc analyses of the three-way interaction of BPV by APOE $\epsilon 4$ carrier status by time in subsets not meeting biomarker criteria for AD revealed no significant relationships with medial temporal volume change (see Supplementary Table 2).

Findings were largely consistent in analyses of diastolic BPV (see Supplementary Results).

Primary findings in hippocampal and entorhinal cortex volume change remained significant and essentially unchanged in sensitivity analyses controlling for average BP, history of smoking, history of dyslipidemia, use 

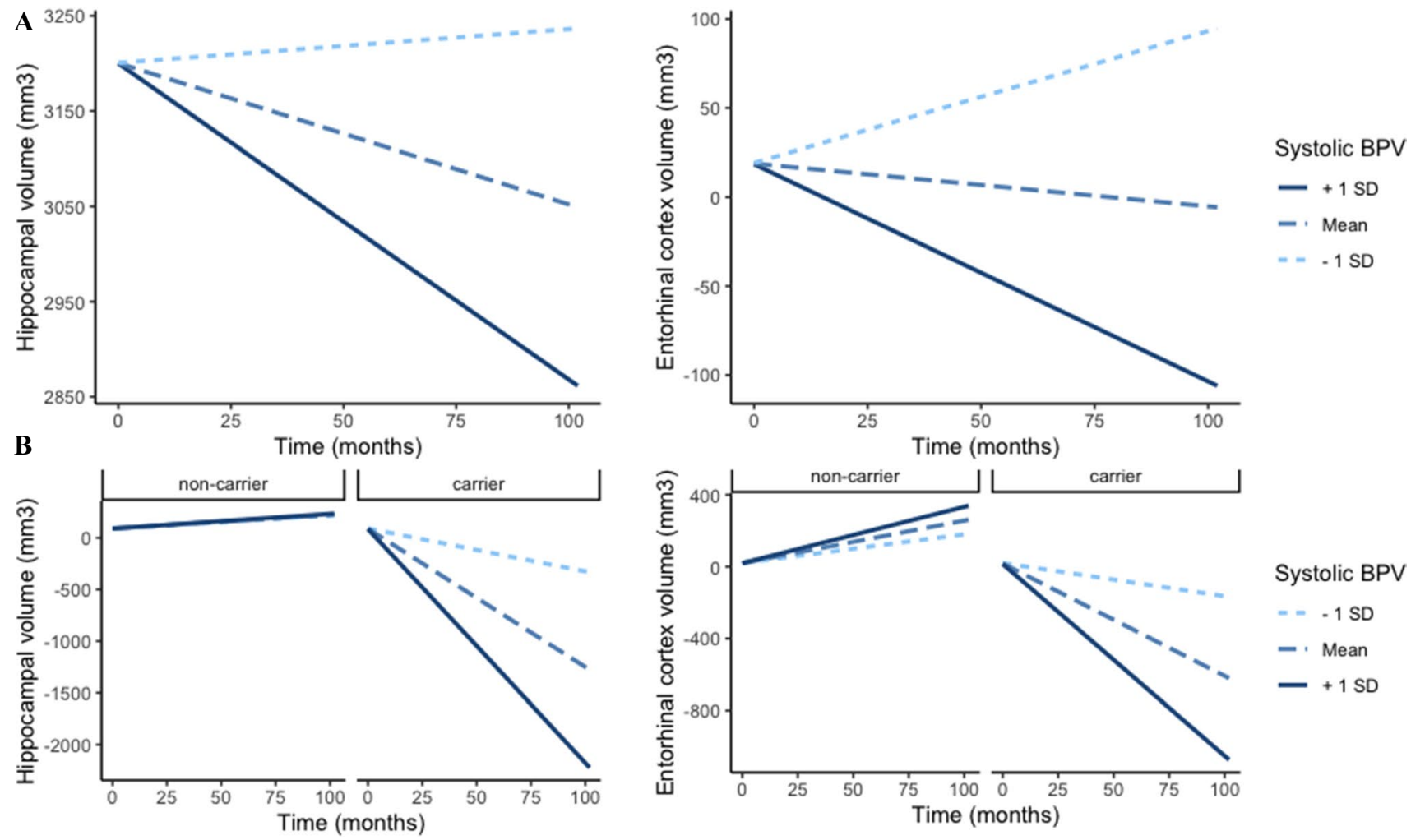

Fig. 1 BPV and medial temporal volumetric change in older adults. Conditional effects of the interaction of A) BPV by time and B) BPV by APOE $€ 4$ carrier status by time on hippocampal and

of antidementia agents, and whole brain volume at MRI (instead of TIV) (Data not shown).

Supplementary analyses revealed a significant interaction of BPV by time on whole brain volume, consistent with one study examining BPV and brain volume change (Ma et al., 2020c). There were no significant interactions with APOE e4 carrier status on whole brain volume (see Supplementary Results).

\section{Discussion}

Findings indicate increased BPV is related to medial temporal volume loss, particularly among APOE $€ 4$ carriers, suggesting a potential interplay between genetic susceptibility to medial temporal pathology and systemic hemodynamic dysregulation. The current investigation confirms prior work linking elevated BPV to hippocampal atrophy (Ma et al., 2020c; Sabayan et al., 2013) and extends findings by identifying the key role of APOE $\epsilon 4$ in determining the relationship between BPV and both hippocampal and entorhinal cortical atrophy. Additionally, the present study identifies relationships between BPV and medial temporal atrophy in older adults with ongoing AD pathophysiology based on entorhinal cortex volume in older adults without history of dementia or stroke. Abbreviations: BPV = blood pressure variability

abnormal levels of both CSF A $\beta$ and Ptau, indicating that links between BPV and medial temporal atrophy are implicated in biomarker-confirmed AD. Study findings provide additional insights into the growing body of evidence that $\mathrm{BPV}$ is associated with $\mathrm{AD}$ and not other neurodegenerative diseases (Lattanzi et al. 2015, 2014a, 2014b).

The link between BPV and volumetric decline was predominantly observed in APOE $\epsilon 4$ carriers, a population known to be at increased risk for medial temporal atrophy (Burggren et al., 2008; Palop \& Mucke, 2011) and AD (Corder et al., 1993). Specifically, an increase of 1 SD in BPV was associated with a $0.27 \%-0.33 \%$ reduction in hippocampal and entorhinal cortex volume per month (3.3\% $-4.0 \%$ per year); in APOE $€ 4$ carriers, volumes declined by $0.67 \%-0.70 \%$ per month $(8.0 \%-8.4 \%$ per year $)$, consistent with prior studies on the association between APOE $€ 4$ and hippocampal volume change (Cohen et al., 2001; Jak et al., 2007; Moffat et al., 2000). APOE $€ 4$ carriers also display breakdown of the blood-brain barrier (Nation et al., 2019) in the hippocampus and parahippocampal cortex before the onset of cognitive impairment and independent of $A \beta$ and tau pathology (Montagne et al., 2020). It has been proposed that chronic high amplitude oscillations in BP may create a repeated "tsunamic effect" in the cerebral parenchyma (Yoo 

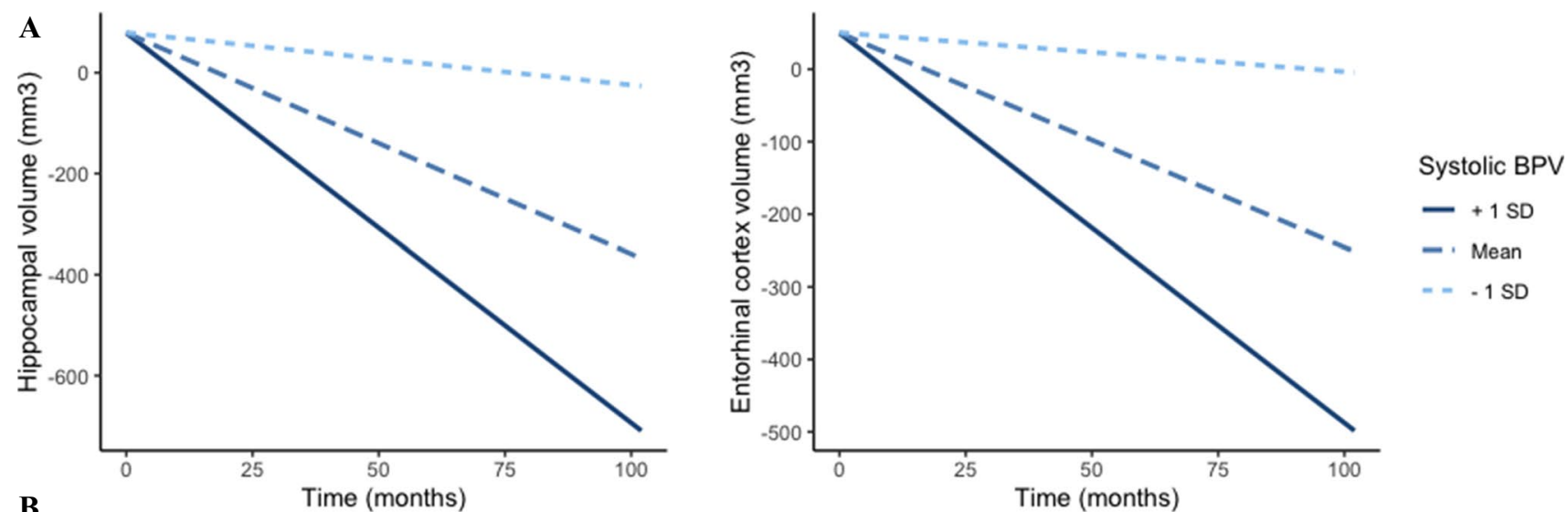

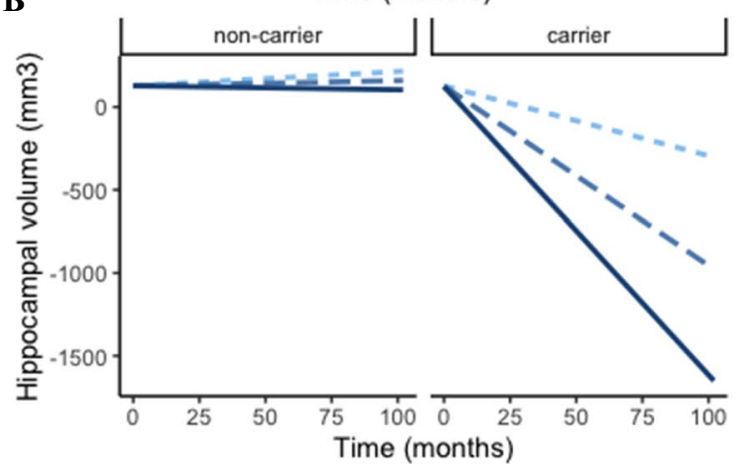

Fig. 2 BPV and medial temporal volumetric change in older adults with AD pathophysiology. Conditional effects of the interaction of A) BPV by time and B) BPV by APOE $€ 4$ carrier status by time on hippocampal and entorhinal cortex volume in older

et al., 2020), distending the arterial walls beyond repair and disrupting the tight junction of the blood-brain barrier. This effect may be especially pronounced in APOE $\epsilon 4$ carriers with genetic neurovascular vulnerability in smaller vascular compartments, where unsteady pulsatile forces may exacerbate a leaky blood-brain barrier (Vikner et al., 2021; Winder et al., 2021) and increase the risk for cerebral small vessel disease. It is striking that the current observations were made in a study sample with limited cerebrovascular disease (Hachinski Ischemic score $\leq 4$ ). More studies of individuals with varying levels of cerebrovascular disease burden will help further elucidate the role BPV may play in blood-brain barrier dysfunction and subsequent neurodegeneration. An alternative possibility is that neurodegeneration in APOE €4 carriers may impact cerebral autonomic control of circulation, potentially driving BP fluctuations (Kitamura et al., 2020). Therefore, causal inference of the current investigation is limited, but the longitudinal design indicating BPV predicts future volumetric change after BPV measurement suggests BPV may play a causal role in brain volume loss in APOE $€ 4$ carriers. Interestingly, BPV was also related to whole brain volume decline, but this was not significantly related to APOE $€ 4$ carrier status. This further highlights the role of BPV in medial temporal atrophy in a population with known regional vulnerability. However, while sensitivity analyses controlling for whole brain volume (instead of TIV) revealed essentially the same pattern of findings, it is important to consider regional volume loss in the context of declining whole brain volume.

In addition to the potential contributions of BPV to cerebrovascular dysfunction, elevated BPV may interfere with vascular clearance mechanisms important for elimination of toxic proteins from the brain (Lattanzi et al., 2018). The observed relationships between BPV and volumetric change were stronger in participant subsets with abnormal levels of CSF A $\beta$ and Ptau and absent in participant subsets not meeting biomarker criteria for $\mathrm{AD}$, suggesting the link between BPV and medial temporal atrophy appears in the context of AD pathophysiology.

Arterial stiffening may amplify BP fluctuations, potentially driving elevated BPV in association with neuronal atrophy (Ma et al., 2020b). While the present investigation was not able to characterize arterial stiffness in the study sample, future work exploring relationships with arterial stiffening may better clarify the contribution of arterial health on brain volume decline. Relatedly, investigating the 
role of BPV in patterns of brain atrophy as a vascular mechanism linking BPV to dementia risk may have therapeutic implications. Emerging evidence suggests that some classes of antihypertensive medication have differential effects on the variability of BP for risk of stroke, independent of average levels (Webb et al., 2010). Large studies adequately powered to investigate treatment effects on BPV, for antihypertensive monotherapy as well as for combination therapy, may lead to additional therapeutic strategies beyond aggressive BP control for the prevention of cognitive impairment. Importantly, BP is a highly modifiable risk factor for dementia (Barnes \& Yaffe, 2011) and even slight changes in BP control may have large public health implications for both cardiovascular and cognitive outcomes (Yaffe, 2019).

The present investigation has a number of strengths. First, by investigating volumetric change after the measurement of $\mathrm{BPV}$, we were able to appreciate the temporal order of the role of BPV and the possibly synergetic effects of BPV and APOE $\epsilon 4$ on volumetric change in brain regions implicated in early AD pathology. Second, to further highlight the possibility of BPV as a vascular risk factor in the context of $\mathrm{AD}$, we utilized participant subsets who were confirmed to have AD pathophysiology based on CSF measurement of $\mathrm{AD}$ biomarkers $\mathrm{A} \beta$ and Ptau.

The study has several noteworthy limitations. Some aspects of BP collection were not explicitly standardized across sites. Additionally, the ADNI database is largely comprised of non-Hispanic White older adults; thus, generalizability of findings to other racial and ethnic groups is limited. There is mixed evidence for whether the relationship between BPV and cerebrovascular disease may differ by race or ethnicity (Brickman et al., 2010; Tully et al., 2020). Studies involving more diverse samples will help to understand potential differences. As part of the inclusionary enrollment criteria for ADNI, the current study did not include older adults with more extensive cerebrovascular disease. Increased BPV has been associated with cerebrovascular damage, which may act as a potential confounder of brain atrophy (Ma et al., 2020a; Sible et al., 2021a; Tully et al., 2020) and contribute to biomarker evidence of AD, possibly independent of APOE $\epsilon 4$. Studies that include samples with varying levels of cerebrovascular disease severity will help clarify the relationship between BPV, cerebrovascular damage, and neurodegeneration.

\section{Conclusions}

Elevated BPV in older adults was related to brain volumetric change over time in regions implicated in $A D$ dementia and pathology, independent of baseline hypertension. APOE $€ 4$ moderated this relationship, suggesting a potentially synergetic effect of both elevated BPV and APOE $\epsilon 4$ on volumetric decline. Finally, patterns of decline were observed to be strongest in individuals with AD pathophysiology, which may implicate BPV as an understudied aspect of vascular contributions to dementia.

Supplementary Information The online version contains supplementary material available at https://doi.org/10.1007/s11682-021-00553-1.

Acknowledgements We would like to thank the participants and their families, investigators, and researchers from the ADNI study.

Authors contributions The listed authors contributed to the present manuscript in the following ways: study concept and design (IJS, DAN), analysis and interpretation of data (IJS, DAN), statistical analyses (IJS), drafting the manuscript (IJS), critical revision of the manuscript (DAN), and final approval of an accountability for the manuscript (IJS, DAN).

Funding The study data analysis was supported by NIH/NIA grants (R01AG064228, R01AG060049, P50AG016573, P01AG052350) and Alzheimer's Association grant AARG-17-532905. Data collection and sharing for this project was funded by the Alzheimer's Disease Neuroimaging Initiative (ADNI) (National Institutes of Health Grant U01 AG024904) and DOD ADNI (Department of Defense award number W81XWH-12-2-0012). ADNI is funded by the National Institute on Aging, the National Institute of Biomedical Imaging and Bioengineering, and through generous contributions from the following: AbbVie, Alzheimer's Association; Alzheimer's Drug Discovery Foundation; Araclon Biotech; BioClinica, Inc.; Biogen; Bristol-Myers Squibb Company; CereSpir, Inc.; Cogstate; Eisai Inc.; Elan Pharmaceuticals, Inc.; Eli Lilly and Company; EuroImmun; F. Hoffmann-La Roche Ltd and its affiliated company Genentech, Inc.; Fujirebio; GE Healthcare; IXICO Ltd.; Janssen Alzheimer Immunotherapy Research \& Development, LLC.; Johnson \& Johnson Pharmaceutical Research \& Development LLC.; Lumosity; Lundbeck; Merck \& Co., Inc.; Meso Scale Diagnostics, LLC.; NeuroRx Research; Neurotrack Technologies; Novartis Pharmaceuticals Corporation; Pfizer Inc.; Piramal Imaging; Servier; Takeda Pharmaceutical Company; and Transition Therapeutics. The Canadian Institutes of Health Research is providing funds to support ADNI clinical sites in Canada. Private sector contributions are facilitated by the Foundation for the National Institutes of Health (www. fnih.org). The grantee organization is the Northern California Institute for Research and Education, and the study is coordinated by the Alzheimer's Therapeutic Research Institute at the University of Southern California. ADNI data are disseminated by the Laboratory for Neuro Imaging at the University of Southern California.

Data availability The datasets generated and/or analyzed during the current study are available in the ADNI database, https://adni.loni. usc.edu.

Code availability All analyses were conducted using R.

\section{Declarations}

Conflicts of interest The authors have no conflicts of interest to disclose.

Ethics approval The present study was conducted in accordance with the Helsinki Declaration and approved at all ADNI sites by local Institutional Review Boards. 
Consent to participate Written informed consent was obtained from all participants.

Consent for publication Not applicable.

Open Access This article is licensed under a Creative Commons Attribution 4.0 International License, which permits use, sharing, adaptation, distribution and reproduction in any medium or format, as long as you give appropriate credit to the original author(s) and the source, provide a link to the Creative Commons licence, and indicate if changes were made. The images or other third party material in this article are included in the article's Creative Commons licence, unless indicated otherwise in a credit line to the material. If material is not included in the article's Creative Commons licence and your intended use is not permitted by statutory regulation or exceeds the permitted use, you will need to obtain permission directly from the copyright holder. To view a copy of this licence, visit http://creativecommons.org/licenses/by/4.0/.

\section{References}

Barnes, D. E., \& Yaffe, K. (2011). The projected effect of risk factor reduction on Alzheimer's disease prevalence. The Lancet Neurology, 10(9), 819-828. https://doi.org/10.1016/S1474-4422(11) $70072-2$

Bittner, T., Zetterberg, H., Teunissen, C. E., Ostlund, R. E., Militello, M., Andreasson, U., et al. (2016). Technical performance of a novel, fully automated electrochemiluminescence immunoassay for the quantitation of $\beta$-amyloid (1-42) in human cerebrospinal fluid. Alzheimer's and Dementia. https://doi.org/10.1016/j.jalz. 2015.09.009

Brickman, A. M., Reitz, C., Luchsinger, J. A., Manly, J. J., Schupf, N., Muraskin, J., et al. (2010). Long-term blood pressure fluctuation and cerebrovascular disease in an elderly cohort. Archives of Neurology, 67(5), 564-569. https://doi.org/10.1001/archneurol. 2010.70

Brown, E. M., Pierce, M. E., Clark, D. C., Fischl, B. R., Iglesias, J. E., Milberg, W. P., et al. (2020). Test-retest reliability of FreeSurfer automated hippocampal subfield segmentation within and across scanners. NeuroImage, 210, 116563. https://doi.org/10.1016/j. neuroimage. 2020.116563

Burggren, A. C., Zeineh, M. M., Ekstrom, A. D., Braskie, M. N., Thompson, P. M., Small, G. W., \& Bookheimer, S. Y. (2008). Reduced cortical thickness in hippocampal sub-regions among cognitively normal apolipoprotein E e4 carriers. NeuroImage, 41(4), 1177-1183. https://doi.org/10.1016/j.neuroimage.2008. 03.039.Reduced

Cho, N., Hoshide, S., Nishizawa, M., Fujiwara, T., \& Kario, K. (2018). Relationship between blood pressure variability and cognitive function in elderly patients with good blood pressure control. American Journal of Hypertension, 31(3), 293-298. https://doi. org/10.1093/ajh/hpx155

Cohen, R. M., Small, C., Lalonde, F., Friz, J., \& Sunderland, T. (2001). Effect of apolipoprotein E genotype on hippocampal volume loss in aging healthy women. Neurology, 57(12), 2223 LP - 2228. https://doi.org/10.1212/WNL.57.12.2223

Corder, E. H., Saunders, A. M., Strittmatter, W. J., Schmechel, D. E., Gaskell, P. C., Small, G. W., et al. (1993). Gene Dose of Apolipoprotein E Type 4 Allele and the Risk of Alzheimer' s Disease in Late Onset Families Published by : American Association for the Advancement of Science Stable URL : http://www.jstor.org/ stable/2882127. Science, 261(5123), 921-923.
D’Agostino, R. B., Wolf, P. A., Belanger, A. J., \& Kannel, W. B. (1994). Stroke risk profile: Adjustment for antihypertensive medication: The Framingham Study. Stroke, 25(1), 40-43. https://doi.org/10. 1161/01.STR.25.1.40

de Heus, R. A. A., Olde Rikkert, M. G. M., Tully, P. J., Lawlor, B. A., \& Claassen, J. A. H. R. (2019). Blood pressure variability and progression of clinical Alzheimer disease. Hypertension, 74, 1172-1180. https://doi.org/10.1161/HYPERTENSIONAHA.119. 13664

Fischl, B. (2012). FreeSurfer. Neuroimage, 62(2), 774-781. https://doi. org/10.1016/j.neuroimage.2012.01.021

Hansson, O., Seibyl, J., Stomrud, E., Zetterberg, H., Trojanowski, J. Q., Bittner, T., et al. (2018). CSF biomarkers of Alzheimer's disease concord with amyloid- $\beta$ PET and predict clinical progression: A study of fully automated immunoassays in BioFINDER and ADNI cohorts. Alzheimer's and Dementia, 14(11), 1470-1481. https://doi.org/10.1016/j.jalz.2018.01.010

Iadecola, C. (2004). Neurovascular regulation in the normal brain and in Alzheimer's disease. Nature Reviews Neuroscience, 5(5), 347-360. https://doi.org/10.1038/nrn1387

Jack, C. R., Bennett, D. A., Blennow, K., Carrillo, M. C., Dunn, B., Haeberlein, S. B., et al. (2018). NIA-AA research framework: Toward a biological definition of Alzheimer's disease. Alzheimer's and Dementia, 14(4), 535-562. https://doi.org/10.1016/j. jalz.2018.02.018

Jak, A. J., Houston, W. S., Nagel, B. J., Corey-Bloom, J., \& Bondi, M. W. (2007). Differential cross-sectional and longitudinal impact of APOE genotype on hippocampal volumes in nondemented older adults. Dementia and Geriatric Cognitive Disorders, 23(6), 382-389. https://doi.org/10.1159/000101340

Kitamura, J., Nagai, M., Ueno, H., Ohshita, T., Kikumoto, M., Toko, M., et al. (2020). The insular cortex, Alzheimer disease pathology, and their effects on blood pressure variability. Alzheimer Disease and Associated Disorders, 34(3), 282-291. https://doi. org/10.1097/WAD.0000000000000340

Lane, C. A., Barnes, J., Nicholas, J. M., Sudre, C. H., Cash, D. M., Parker, T. D., et al. (2019). Associations between blood pressure across adulthood and late-life brain structure and pathology in the neuroscience substudy of the 1946 British birth cohort (Insight 46): An epidemiological study. The Lancet Neurology, 18(10), 942-952. https://doi.org/10.1016/S1474-4422(19) 30228-5

Lattanzi, S., Luzzi, S., Provinciali, L., \& Silvestrini, M. (2014a). Blood pressure variability predicts cognitive decline in Alzheimer's disease patients. Neurobiology of Aging, 35(10), 2282-2287. https:// doi.org/10.1016/j.neurobiolaging.2014.04.023

Lattanzi, S., Luzzi, S., Provinciali, L., \& Silvestrini, M. (2015). Blood pressure variability in alzheimer's disease and frontotemporal dementia: The effect on the rate of cognitive decline. Journal of Alzheimer's Disease, 45(2), 387-394. https://doi.org/10.3233/ JAD-142532

Lattanzi, S., Vernieri, F., \& Silvestrini, M. (2018). Blood pressure variability and neurocognitive functioning. Journal of Clinical Hypertension, 20(4), 645-647. https://doi.org/10.1111/jch.13232

Lattanzi, S., Viticchi, G., Falsetti, L., Buratti, L., Luzzi, S., Provinciali, L., \& Silvestrini, M. (2014b). Visit-to-visit blood pressure variability in Alzheimer disease. Alzheimer Disease and Associated Disorders, 28(4), 347-351. https://doi.org/10.1097/WAD.00000 00000000040

Ma, Y., Song, A., Viswanathan, A., Blacker, D., Vernooij, M. W., Hofman, A., \& Papatheodorou, S. (2020a). Blood pressure variability and cerebral small vessel disease: A systematic review and metaanalysis of population-based cohorts. Stroke, 51(1), 82-89. https:// doi.org/10.1161/STROKEAHA.119.026739

Ma, Y., Tully, P. J., Hofman, A., \& Tzourio, C. (2020b). Blood pressure variability and dementia: A state-of-the art review. American 
Journal of Hypertension, 33(12), 1059-1066. https://doi.org/10. 1093/ajh/hpaa119

Ma, Y., Yilmaz, P., Bos, D., Blacker, D., Viswanathan, A., Ikram, M. A., et al. (2020c). Blood pressure variation and subclinical brain disease. Journal of the American College of Cardiology, 75(19), 2387-2399. https://doi.org/10.1016/j.jacc.2020.03.043

Moffat, S. D., Szekely, C. A., Zonderman, A. B., Kabani, N. J., \& Resnick, S. M. (2000). Longitudinal change in hippocampal volume as a function of apolipoprotein E genotype. Neurology, 55(1), 134 LP - 136. https://doi.org/10.1212/WNL.55.1.134

Montagne, A., Nation, D. A., Sagare, A. P., Barisano, G., Sweeney, M. D., Chakhoyan, A., et al. (2020). APOE4 leads to early bloodbrain barrier dysfunction predicting human cognitive decline. Nature, 581(7806), 71-76. https://doi.org/10.1038/s41586-0202247-3.APOE4

Nagai, M., Dote, K., Kato, M., Sasaki, S., Oda, N., Kagawa, E., et al. (2017). Visit-to-visit blood pressure variability and Alzheimer's disease: Links and risks. Journal of Alzheimer's Disease, 59(2), 515-526. https://doi.org/10.3233/JAD-161172

Nation, D. A., Edmonds, E. C., Bangen, K. J., Delano-Wood, L., Scanlon, B. K., Han, S. D., et al. (2015). Pulse pressure in relation to tau-mediated neurodegeneration, cerebral amyloidosis, and progression to dementia in very old adults. JAMA Neurology, 72(5), 546-553. https://doi.org/10.1001/jamaneurol.2014.4477

Nation, D. A., Sweeney, M. D., Montagne, A., Sagare, A. P., D’Orazio, L. M., Pachicano, M., et al. (2019). Blood-brain barrier breakdown is an early biomarker of human cognitive dysfunction. Nature Medicine, 25(2), 270-276. https://doi.org/10.1038/s41591018-0297-y.Blood-brain

Palop, J. J., \& Mucke, L. (2011). Amyloid- $\beta$ induced neuronal dysfunction in Alzheimer's disease: From synapses toward neural networks. Nature Neuroscience, 13(7), 812-818. https://doi.org/ 10.1038/nn.2583.Amyloid-

Petersen, R. C., Aisen, P. S., Beckett, L. A., Donohue, M. C., Gamst, A. C., Harvey, D. J., et al. (2010). Alzheimer's disease neuroimaging initiative (ADNI): Clinical characterization. Neurology, 74(3), 201-209. https://doi.org/10.1212/WNL.0b013e3181cb3e25

R Core Team. (2018). R: A language and environment for statistical computing.

Reuter, M., Schmansky, N. J., Rosas, H. D., \& Fischl, B. (2012). Within-subject template estimation for unbiased longitudinal image analysis. NeuroImage, 61(4), 1402-1418. https://doi.org/ 10.1016/j.neuroimage.2012.02.084

Rothwell, P. M., Howard, S. C., Dolan, E., O’Brien, E., Dobson, J. E., Dahlöf, B., et al. (2010). Prognostic significance of visit-to-visit variability, maximum systolic blood pressure, and episodic hypertension. The Lancet, 375(9718), 895-905. https://doi.org/10.1016/ S0140-6736(10)60308-X

Rouch, L., Cestac, P., Sallerin, B., Piccoli, M., Benattar-Zibi, L., Bertin, P., et al. (2020). Visit-to-visit blood pressure variability is associated with cognitive decline and incident dementia: The S.AGES cohort. Hypertension, 76, 1280-1288. https://doi.org/ 10.1161/HYPERTENSIONAHA.119.14553

Sabayan, B., Wijsman, L. W., Foster-Dingley, J. C., Stott, D. J., Ford, I., Buckley, B. M., et al. (2013). Association of visit-to-visit variability in blood pressure with cognitive function in old age: Prospective cohort study. BMJ (Online), 347(7919). https://doi.org/ 10.1136/bmj.f4600

Saykin, A. J., Shen, L., Foroud, T. M., Potkin, S. G., Swaminathan, S., Kim, S., et al. (2010). Alzheimer's disease neuroimaging Initiative biomarkers as quantitative phenotypes: Genetics core aims, progress, and plans. Alzheimer's and Dementia, 6(3), 265-273. https://doi.org/10.1016/j.jalz.2010.03.013

Schneider, J. A., Arvanitakis, Z., Bang, W., \& Bennett, D. A. (2007). Mixed brain pathologies account for most dementia cases in community-dwelling older persons. Neurology, 69, 2197-2204. https://doi.org/10.1212/01.wnl.0000307675.38908.39

Seibyl, J., Shaw, L. M., Blennow, K., Widmann, M., Corradini, V., Wahl, S., et al. (2017). Amyloid-PET concordance of Elecsys ${ }^{\circledR}$ CSF biomarker immunoassays for Alzheimer's disease. Alzheimer's \& Dementia, 13(7), P199-P200. https://doi.org/10.1016/j. jalz.2017.07.062

Shaw, L. M., Fields, L., Korecka, M., Waligórska, T., Trojanowski, J. Q., Allegranza, D., et al. (2016). Method comparison of AB(1-42) measured in human cerebrospinal fluid samples by liquid chromatography-tandem mass spectrometry, the INNO-BIA AlzBio3 assay, and the Elecsys ${ }^{\circledR}$ B-Amyloid(1-42) assay. Alzheimer's \& Dementia, 12(7), P668. https://doi.org/10.1016/j.jalz.2016.06. 1513

Shaw, L. M., Waligorska, T., Fields, L., Korecka, M., Figurski, M., Trojanowski, J. Q., et al. (2018). Derivation of cutoffs for the Elecsys ${ }^{\circledR}$ amyloid $\beta(1-42)$ assay in Alzheimer's disease. Alzheimer's and Dementia: Diagnosis, Assessment and Disease Monitoring, 10, 698-705. https://doi.org/10.1016/j.dadm.2018.07.002

Sible, I. J., Bangen, K. J., Blanken, A. E., Ho, J. K., \& Nation, D. A. (2021a). Antemortem visit-to-visit blood pressure variability predicts cerebrovascular lesion burden in autopsy-confirmed Alzheimer's disease. Journal of Alzheimer's Disease.

Sible, I. J., Nation, D. A., \& Alzheimer's Disease Neuroimaging Initiative. (2020). Long-term blood pressure variability across the clinical and biomarker spectrum of Alzheimer's disease. Journal of Alzheimer's Disease, 77(4), 1655-1669. https://doi.org/10.3233/ JAD-200221

Sible, I. J., Yew, B., Dutt, S., Bangen, K. J., Li, Y., Nation, D. A., \& Alzheimer's Disease Neuroimaging Initiative. . (2021b). Visitto-visit blood pressure variability and regional cerebral perfusion decline in older adults. Neurobiology of Aging, 105, 57-63. https://doi.org/10.1016/j.neurobiolaging.2021.04.009

Tully, P. J., Yano, Y., Launer, L. J., Kario, K., Nagai, M., Mooijaart, S. P., et al. (2020). Association between blood pressure variability and cerebral small-vessel disease: A systematic review and metaanalysis. Journal of the American Heart Association, 9(1). https:// doi.org/10.1161/JAHA.119.013841

Vikner, T., Eklund, A., Karalija, N., Malm, J., Riklund, K., Lindenberger, U., et al. (2021). Cerebral arterial pulsatility is linked to hippocampal microvascular function and episodic memory in healthy older adults. Journal of Cerebral Blood Flow and Metabolism. https://doi.org/10.1177/0271678X20980652

Webb, A. J., Fischer, U., Mehta, Z., \& Rothwell, P. M. (2010). Effects of antihypertensive-drug class on interindividual variation in blood pressure and risk of stroke: A systematic review and metaanalysis. The Lancet, 375(9718), 906-915. https://doi.org/10. 1016/S0140-6736(10)60235-8

Winder, N. R., Reeve, E. H., \& Walker, A. E. (2021). Large artery stiffness and brain health: Insights from animal models. American Journal of Physiology - Heart and Circulatory Physiology, 320(1), H424-H431. https://doi.org/10.1152/AJPHEART.00696. 2020

Wright, J. T., Williamson, J. D., Whelton, P. K., Snyder, J. K., Sink, K. M., Rocco, M. V., et al. (2015). A randomized trial of intensive versus standard blood-pressure control. New England Journal of Medicine, 373(22), 2103-2116. https://doi.org/10.1056/NEJMo a1511939

Yaffe, K. (2019). Prevention of cognitive impairment with intensive systolic blood pressure control. JAMA, 321(6), 548-549.

Yano, Y. (2017). Visit-to-visit blood pressure variability - What is the current challenge? American Journal of Hypertension, 30(2), 112-114. https://doi.org/10.1093/ajh/hpw124

Yoo, J. E., Shin, D. W., Han, K., Kim, D., Lee, S. P., Jeong, S. M., et al. (2020). Blood pressure variability and the risk of dementia: 
A nationwide cohort study. Hypertension, 75(4), 982-990. https:// doi.org/10.1161/HYPERTENSIONAHA.119.14033

Zlokovic, B. V. (2011). Neurovascular pathways to neurodegeneration in Alzheimer's disease and other disorders. Nature Reviews Neuroscience, 12(12), 723-738. https://doi.org/10.1038/nrn3114
Publisher's note Springer Nature remains neutral with regard to jurisdictional claims in published maps and institutional affiliations. 